BMJ Open

Diabetes

Research

\& Care

\title{
The reliability of in-hospital diagnoses of diabetes mellitus in the setting of an acute myocardial infarction
}

\author{
Suzanne V Arnold, ${ }^{1}$ Kasia J Lipska, ${ }^{2}$ Silvio E Inzucchi, ${ }^{2}$ Yan Li, ${ }^{3}$ Philip G Jones, ${ }^{3}$ \\ Darren K McGuire, ${ }^{4}$ Abhinav Goyal, ${ }^{5}$ Joshua M Stolker, ${ }^{6}$ Marcus Lind, ${ }^{7}$ \\ John A Spertus, ${ }^{1}$ Mikhail Kosiborod ${ }^{1}$
}

To cite: Arnold SV, Lipska KJ, Inzucchi SE, et al. The reliability of in-hospital diagnoses of diabetes mellitus in the setting of an acute myocardial infarction. BMJ Open Diabetes Research and Care 2014;2: 000046. doi:10.1136/bmjdrc-2014000046

- Additional material is available. To view please visit the journal (http://dx.doi.org/ 10.1136/bmjdrc-2014-

000046)

Received 18 July 2014 Revised 25 September 2014 Accepted 21 October 2014

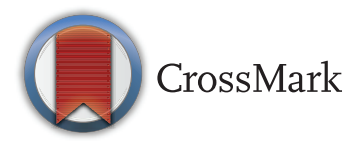

For numbered affiliations see end of article.

Correspondence to Suzanne V Arnold; sva1024@yahoo.com

\section{ABSTRACT}

Objective: Incident diabetes mellitus (DM) is important to recognize in patients with acute myocardial infarction (AMI). To develop an efficient screening strategy, we explored the use of random plasma glucose (RPG) at admission and fasting plasma glucose (FPG) to select patients with AMI for glycosylated hemoglobin (HbA1c) testing.

Design, setting, andparticipants: Prospective registry of 1574 patients with AMI not taking glucoselowering medication from 24 US hospitals. All patients had $\mathrm{HbA1c}$ measured at a core laboratory and admission RPG and $\geq 2$ FPGs recorded during hospitalization. We examined potential combinations of $\mathrm{RPG}$ and FPG and compared these with $\mathrm{HbA} 1 \mathrm{c} \geq 6.5 \%$ - considered the gold standard for DM diagnosis in these analyses.

Results: An RPG $>140 \mathrm{mg} / \mathrm{dL}$ or $\mathrm{FPG} \geq 126 \mathrm{mg} / \mathrm{dL}$ had high sensitivity for DM diagnosis. Combining these into a screening protocol (if admission RPG $>140$, check $\mathrm{HbA1C}$; or if $F P G \geq 126$ on a subsequent day, check $\mathrm{HbA1c}$ ) led to $\mathrm{HbA} 1 \mathrm{c}$ testing in $50 \%$ of patients and identified $86 \%$ with incident DM (number needed to screen (NNS)=3.3 to identify 1 case of DM; vs $\mathrm{NNS}=5.6$ with universal $\mathrm{HbA1c}$ screening). Alternatively, using an RPG $>180$ led to $\mathrm{HbA1c}$ testing in $40 \%$ of patients with AMI and identified $82 \%$ of DM (NNS=2.7).

Conclusions: We have established two potential selective screening methods for DM in the setting of AMI that could identify the vast majority of incident DM by targeted screening of $40-50 \%$ of patients with AMI with HbA1c testing. Using these methods may efficiently identify patients with AMI with DM so that appropriate education and treatment can be promptly initiated.

Type 2 diabetes mellitus (DM) is present in approximately one-third of patients hospitalized with an acute myocardial infarction $(\mathrm{AMI})^{12}$ and is associated with an increased risk of short-term and long-term mortality. ${ }^{3} 4$ Furthermore, approximately one-quarter of patients with coronary artery disease have undiagnosed DM. ${ }^{5}{ }^{6}$ Recognizing these patients is important, as identification of

\section{Key messages}

- In a large, multicenter US cohort, we identified two potential selective screening methods for the detection of diabetes in the setting of an acute myocardial infarction using blood glucose levels collected as part of routine clinical care.

- These screening protocols required testing glycosylated hemoglobin in only $40-50 \%$ of patients with myocardial infarction and yet identified the vast majority of those with incident diabetes-an attractive strategy for clinical and economic reasons.

- Since patients with both myocardial infarction and diabetes are at high risk for recurrent cardiovascular events and the microvascular complications of diabetes, identification of patients with diabetes during the acute hospitalization is important, so these individuals can be targeted for more intensive lifestyle and medical interventions.

metabolic abnormalities at the time of AMI may allow for initiation of lifestyle modification, DM education, pharmacological therapy prior to discharge or referral to endocrinologists for outpatient follow-up. In addition, the presence of DM informs clinical decision making for cardiovascular care, such as revascularization options and selection and titration of selected cardiovascular medications. Introducing care strategies at the time of discharge may have the greatest likelihood of being implemented by the patient. ${ }^{78}$

Diagnosing DM during an AMI has potential challenges, however, as adrenergic and other counter-regulatory changes that occur with an AMI are associated with substantial dysregulation in glucose levels. ${ }^{9}$ For this reason, oral glucose tolerance testing, which is more reliable than glucose levels in the setting of AMI, had been previously recommended for screening of DM among patients with AMI. ${ }^{10}{ }^{11}$ However, this method of screening can be inconvenient and is rarely 
used clinically in the USA, particularly during acute hospitalizations. Recently, glycosylated hemoglobin (HbA1c) testing, which is generally unaffected by transient perturbations in blood glucose and does not require fasting samples, has emerged as a new and more convenient standard for the diagnosis of DM.

While screening all patients with AMI with HbAlc is a potentially attractive strategy to detect incident DM, more selective strategies can identify the vast majority of patients with $\mathrm{DM}$ and be more cost-efficient. ${ }^{12}$ In addition to their clinical implications, identifying blood glucose cut-points that can accurately identify patients with DM would also be useful for research purposes; for example, in data sets where a surrogate definition of DM is needed due to missing or unmeasured HbAlc values. As such, we used a multicenter AMI registry with core laboratory-assessed HbAlc to explore different potential definitions of DM based on various fasting and random glucose cut-points, to determine if there were particular combinations of these that would be a useful initial screen for DM during AMI.

\section{METHODS}

\section{Study population and protocol}

Details of the Translational Research Investigating Underlying disparities in acute Myocardial infarction Patients' Health status (TRIUMPH) registry have been previously described. ${ }^{13}$ In brief, eligible patients had biomarker evidence of myocardial necrosis and additional clinical evidence supporting the diagnosis of an AMI. Baseline data were obtained through chart abstraction and a structured interview. All enrolled patients were asked to participate in a laboratory substudy, and consenting patients had a fasting blood specimen collected just prior to discharge, which was analyzed by a core laboratory (Clinical Reference Laboratory, Lenexa, Kansas, USA). Blood was analyzed for HbAlc, glucose and insulin levels, and lipids, the results of which were blinded to clinicians. Core laboratory HbAlc was assessed with the Bio-Rad VARIANT II assay, which is certified by the National Glycohemoglobin Standardization Program and standardized to the Diabetes Control and Complications Trial reference assay. Chart-derived HbA1c levels were not used in this study to ensure consistency and reliability of the HbA1c diagnosis of DM.

Laboratory values drawn for clinical purposes were also recorded, which included a random glucose (random plasma glucose, RPG) on admission and up to three chart values of fasting glucose (fasting plasma glucose, FPG), which were measured on venous plasma samples (ie, capillary blood glucose measurements were not collected). If a patient had $>3$ FPGs analyzed during the hospitalization, the first three were recorded. No other RPG values (beyond the admission value) were recorded for the hospitalization. To ensure we had a consistent patient population, all included patients were required to have an HbAlc analyzed at the core laboratory and chart-derived values for an RPG on admission and $\geq 2$ FPGs. As glucose-lowering medications could interfere with the association between HbAlc level and glucose levels, patients who were on glucose-lowering medications at admission were excluded. Use of inpatient glucose-lowering medications was not considered. Each participating hospital obtained Institutional Research Board approval, and all patients provided written informed consent.

\section{Analytic plan}

We evaluated various definitions for DM, based on FPG and RPG (at admission), using HbAlc $\geq 6.5 \%$ as the gold standard for diagnosis per the American Diabetes Association guidelines. ${ }^{14}$ Potential definitions included the following: (A) RPG $>140 \mathrm{mg} / \mathrm{dL}$; (B) RPG $>180 \mathrm{mg}$ / dL; (C) RPG $\geq 200 \mathrm{mg} / \mathrm{dL}$; (D) $\geq 1$ FPG levels $\geq 126 \mathrm{mg} /$ dL; (E) $\geq 2$ FPG levels $\geq 126 \mathrm{mg} / \mathrm{dL} ;$ (F) $\geq 1$ FPG levels $>140 \mathrm{mg} / \mathrm{dL} ;(\mathrm{G}) \geq 2$ FPG levels $>140 \mathrm{mg} / \mathrm{dL} ;(\mathrm{H}) \geq 1$ FPG $\geq 126 \mathrm{mg} / \mathrm{dL}$ and $\mathrm{RPG} \geq 200 \mathrm{mg} / \mathrm{dL}$; and (I) $\geq 1$ FPG $>140 \mathrm{mg} / \mathrm{dL}$ and $\mathrm{RPG} \geq 200 \mathrm{mg} / \mathrm{dL}$. The various thresholds for FPG and RPG were selected based on prior guidelines ${ }^{1415}$ and on prior studies examining the association of stress hyperglycemia with mortality. ${ }^{9}$ For each of these definitions, we calculated the sensitivity, specificity, positive-predictive (PPV) and negativepredictive values (NPV), and accuracy (\% of true results (i.e., (true positives+true negatives)/total)) for the diagnosis of DM, as defined by an HbA1c $\geq 6.5 \%$. We also examined the impact on screening volume and DM recognition using two-step screening processes, which included an initial screen based on RPG at admission (with 2 thresholds, per definitions A or B) followed by screens based on FPGs on subsequent days of hospitalization (per definition E). Finally, in a sensitivity analysis, as confirmatory HbAlc testing is recommended prior to a diagnosis of DM, we examined the screening strategies among patients with an HbAlc $\geq 6.5 \%$ (by our core laboratory) and an additional HbA1c laboratory value (drawn at the local hospital for clinical purposes) of $\geq 6.5 \%$. All analyses were conducted using SAS V.9.4 (SAS Institute, Inc, Cary, North Carolina, USA).

\section{RESULTS \\ Study population}

Among 4340 patients with AMI enrolled in TRIUMPH, 952 were excluded due to the use of glucose-lowering medications, 1156 did not consent to the laboratory substudy and thus did not have core HbAlc levels, and 658 were missing the minimum number of fasting and/or random glucose measurements. The final analytic cohort thus included 1574 patients with complete data available. Patients who were excluded due to nonparticipation in the laboratory substudy or missing chart data were generally similar to those included in the study, although excluded patients were slightly older, more likely to be non-white, and to present with a 
Table 1 Baseline demographic and clinical characteristics

\begin{tabular}{lc}
\hline & $\mathrm{n}=1574$ \\
\hline Age (year) & $58.0 \pm 12.5$ \\
Male & $70.1 \%$ \\
White & $71.9 \%$ \\
Married & $55.3 \%$ \\
High school education & $82.3 \%$ \\
Body mass index (kg/m ${ }^{2}$ ) & $29.0 \pm 5.9$ \\
Current smoking & $44.8 \%$ \\
Hypertension & $61.7 \%$ \\
Prior myocardial infarction & $17.9 \%$ \\
Prior angioplasty & $17.7 \%$ \\
Prior bypass surgery & $8.6 \%$ \\
Prior stroke & $4.1 \%$ \\
Chronic lung disease & $6.4 \%$ \\
ST-elevation myocardial infarction & $50.1 \%$ \\
Left ventricular ejection fraction $<40 \%$ & $17.4 \%$ \\
In-hospital coronary angiogram & $96.4 \%$ \\
In-hospital percutaneous coronary & $70.7 \%$ \\
intervention & \\
In-hospital coronary artery bypass surgery & $9.2 \%$ \\
HbA1c (\%) & $6.1 \pm 1.4$ \\
HbA1c $\geq 6.5 \%$ & $17.9 \%$ \\
Insulin level ( $\mu$ lU/mL) & $16.7 \pm 24.5$ \\
Total cholesterol (mg/dL) & $156.7 \pm 37.2$ \\
Triglycerides (mg/dL) & $154.8 \pm 104.7$ \\
High-density lipoprotein cholesterol (mg/dL) & $39.9 \pm 10.5$ \\
Low-density lipoprotein cholesterol (mg/dL) & $96.0 \pm 31.2$ \\
\hline HbA1c, glycosylated hemoglobin. & \\
& \\
\hline & \\
\hline & \\
\hline
\end{tabular}

non-ST-elevation AMI (eTable 1). However, among patients with some (but not complete) laboratory values available ( $n=1136$ for HbA1c; $n=1012$ for FPG), HbA1c levels and FPG levels were qualitatively similar between those excluded and included in the analytic sample.

The demographic, clinical, and metabolic factors of the analytic population are shown in table 1. Overall, the mean age of the patients included in the final analytic cohort was 58 years, $70 \%$ were male, $72 \%$ were white, and $50 \%$ presented with ST-elevation AMI. Among the 1574 patients with AMI not on glucoselowering medications, mean $\mathrm{HbAlc}$ was $6.1 \% \pm 1.4 \%$, and $18 \%$ had $\mathrm{HbAlc} \geq 6.5 \%$. The mean of the highest FPG during the hospitalization was $128 \mathrm{mg} / \mathrm{dL}$. The percentages of patients meeting each proposed definition of DM based on RPG and FPG are shown in table 2.

\section{Sensitivity, specificity, PPV, and NPV of different diagnostic approaches}

The sensitivity, specificity, NPV, PPV, and accuracy of the 9 potential diagnoses for $\mathrm{DM}$ are shown in table 2 . Definitions A (RPG $>140 \mathrm{mg} / \mathrm{dL}), \quad \mathrm{D} \quad(\geq 1 \quad \mathrm{FPG}$ $\geq 126 \mathrm{mg} / \mathrm{dL}$ ), and F ( $\geq 1 \mathrm{FPG} \geq 140 \mathrm{mg} / \mathrm{dL}$ ) all had reasonably high sensitivity for an $\mathrm{HbA1c} \geq 6.5 \%$, and not exceeding these thresholds had high NPVs for excluding DM, and could be used for initial screening, as they would result in very few false-negatives. Using definition $\mathrm{D}$ (the highest sensitivity among definitions) alone as a trigger for $\mathrm{HbAlc}$ testing would require $\mathrm{HbA1c}$ testing of $36 \%$ of the AMI population and identify $78 \%$ of patients with DM, with a number needed to screen of 2.6 patients with AMI to identify 1 case of DM.

In order to maximize the number of patients with DM identified with a strategy that would fit with clinical care, we combined an initial screen with RPG on admission with screens by FPG on subsequent hospitalization days (figure 1). The first strategy (strategy 1; figure 1A) uses an RPG on presentation of $>140 \mathrm{mg} / \mathrm{dL}$ as the initial trigger for HbAlc check. If on-arrival RPG was $<140 \mathrm{mg} / \mathrm{dL}$, then if an FPG is $\geq 126 \mathrm{mg} / \mathrm{dL}$ on a subsequent hospitalization day, HbAlc testing would be triggered. In our cohort, this strategy would require HbAlc testing of $50.2 \%$ of the patients with AMI and identify $86 \%$ of patients with DM in the cohort, with an NPV of $95 \%$. The 39 patients who met diagnostic DM criteria by HbAlc who would not have been identified with this screening protocol had a median HbAlc of $6.8 \%$ (IQR

Table 2 Reliability of different potential definitions of diabetes, using $\mathrm{HbA} 1 \mathrm{c} \geq 6.5 \%$ as the gold standard

\begin{tabular}{|c|c|c|c|c|c|c|}
\hline Potential definition & $\begin{array}{l}\text { Patients meeting } \\
\text { criteria }(\%)^{*}\end{array}$ & $\begin{array}{l}\text { Sensitivity } \\
(\%)\end{array}$ & $\begin{array}{l}\text { Specificity } \\
(\%)\end{array}$ & $\begin{array}{l}\text { PPV } \\
\text { (\%) }\end{array}$ & $\begin{array}{l}\text { NPV } \\
(\%)\end{array}$ & $\begin{array}{l}\text { Accuracy } \dagger \\
(\%)\end{array}$ \\
\hline (A) RPG $>140 \mathrm{mg} / \mathrm{dL}$ & 33.8 & 70.9 & 74.3 & 37.6 & 92.1 & 73.7 \\
\hline (B) $\mathrm{RPG}>180 \mathrm{mg} / \mathrm{dL}$ & 14.3 & 50.4 & 93.6 & 63.1 & 89.6 & 85.8 \\
\hline (C) $R P G \geq 200 \mathrm{mg} / \mathrm{dL}$ & 10.8 & 43.6 & 96.4 & 72.4 & 88.7 & 86.9 \\
\hline (D) $\geq 1 \mathrm{FPG}$ levels $\geq 126 \mathrm{mg} / \mathrm{dL}$ & 36.2 & 78.4 & 73.0 & 38.8 & 93.9 & 74.0 \\
\hline (E) $\geq 2$ FPG levels $\geq 126 \mathrm{mg} / \mathrm{dL}$ & 17.0 & 58.9 & 92.2 & 62.2 & 91.1 & 86.2 \\
\hline (F) $\geq 1$ FPG levels $>140 \mathrm{mg} / \mathrm{dL}$ & 23.1 & 67.0 & 86.5 & 51.9 & 92.3 & 83.0 \\
\hline (G) $\geq 2$ FPG levels $>140 \mathrm{mg} / \mathrm{dL}$ & 10.0 & 42.2 & 97.1 & 75.8 & 88.5 & 87.2 \\
\hline $\begin{array}{l}\text { (H) } \geq 1 F P G \geq 126 \text { and } R P G \\
\text { (I) } \geq 200 \mathrm{mg} / \mathrm{dL}\end{array}$ & 8.6 & 40.4 & 98.4 & 84.4 & 88.3 & 88.0 \\
\hline $\begin{array}{l}\text { (J) } \geq 1 F P G>140 \text { and } R P G \\
(K) \geq 200 \mathrm{mg} / \mathrm{dL}\end{array}$ & 7.7 & 37.9 & 98.9 & 88.4 & 88.0 & 88.0 \\
\hline
\end{tabular}

*Prevalence of diabetes per $\mathrm{HbA} 1 \mathrm{c}$ screening $=17.9 \%$.

$\dagger$ Accuracy $=\%$ of true results (ie, (true positives+true negatives)/total).

FPG, fasting plasma glucose; HbA1c, glycosylated hemoglobin; NPV, negative-predictive value; PPV, positive-predictive value; RPG, random plasma glucose (collected at admission). 
Figure 1 Schematic of screening program for diabetes mellitus (DM) during acute myocardial infarction (AMI). Each strategy uses a random plasma glucose (RPG) at admission greater than a defined threshold, or fasting plasma glucose (FPG) $\geq 126 \mathrm{mg} / \mathrm{dL}$ on any subsequent day, to trigger glycosylated hemoglobin $(\mathrm{HbA1c})$ testing.

(A) Strategy 1: the results using a cut-point for admission RPG $>140 \mathrm{mg} / \mathrm{dL}$ by FPG for screening. (B) Strategy 2: the results using a cut-point for admission glucose of $>180 \mathrm{mg} / \mathrm{dL}$ for screening. Strategy 1 has a sensitivity $86 \%$, specificity $58 \%$, positive-predictive value (PPV) $31 \%$, negative-predictive value (NPV) 95\%, and accuracy63\%. Strategy 2 has a sensitivity $82 \%$, specificity 69\%, PPV 37\%, NPV $95 \%$, and accuracy $71 \%$.

\section{A Strategy 1}

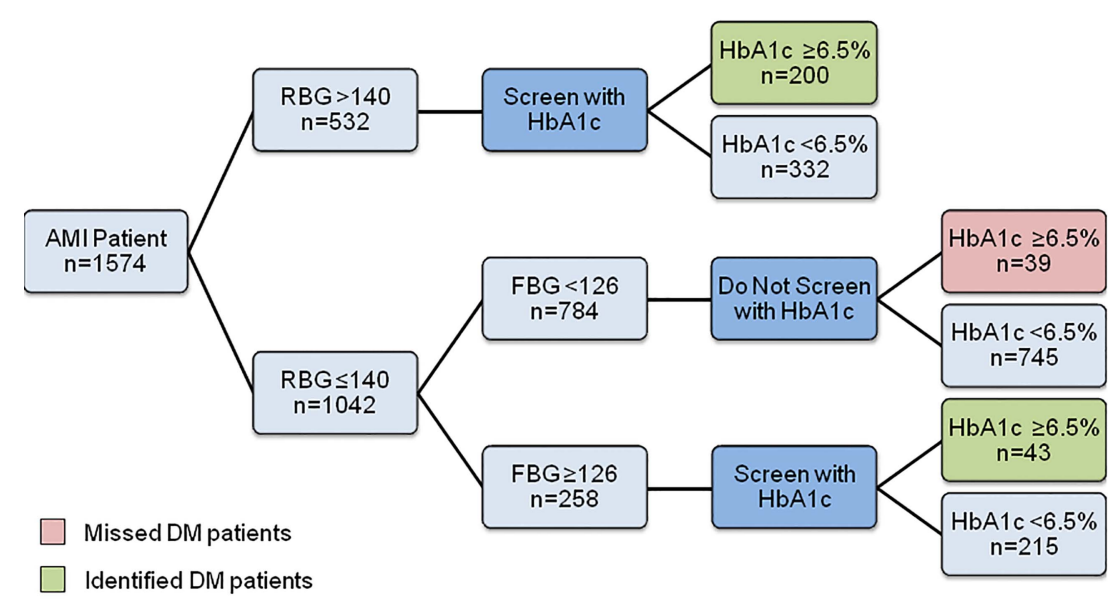

B Strategy 2

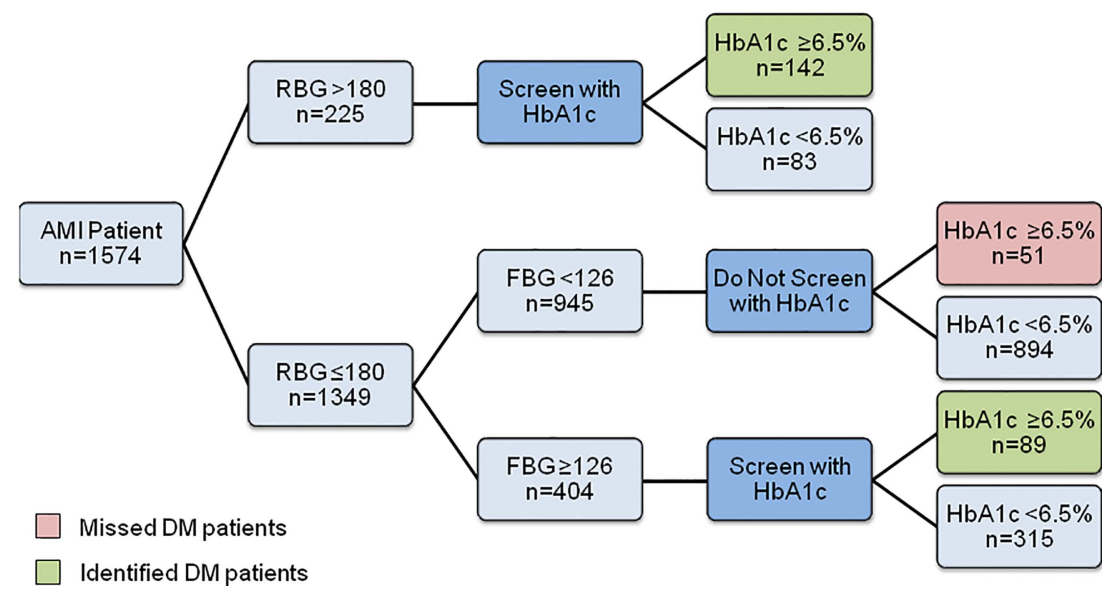

6.5-6.9\%). Alternatively, using an RPG cut-point of $>180 \mathrm{mg} / \mathrm{dL}$ to trigger an HbA1c test (strategy 2; figure 1B) would require $\mathrm{HbA1c}$ testing of $40 \%$ of the AMI population and identify $82 \%$ of patients with DM, with an NPV of $95 \%$. The 51 remaining, unrecognized patients with DM had a median HbAlc of $6.8 \%$ (IQR 6.5-6.9\%). Comparing these two-step screening methods, strategy 1 would screen an additional $10 \%$ of AMI population to identify $4 \%$ more of the patients with DM. The number needed to screen to identify 1 case of DM would be 3.3 with strategy $1,2.7$ with strategy 2 , and 5.6 with universal screening (ie, screening all patients with AMI using $\mathrm{HbA1c}$ ). In the sensitivity analysis examining only patients who had a core and chart HbAlc level $\geq 6.5 \% \quad(n=164)$, strategy 1 would identify 154 patients $(94 \%)$ of those who met criteria for DM, and strategy 2 would identify 153 patients (93\%).

In the analyses designed to identify the ideal combination of blood glucose levels for diagnosing DM (ie, for research purposes in the absence of measured $\mathrm{HbAlc}$ levels), the most accurate estimation of the presence of DM using only RPG and FPG was similar for all definitions except A (RPG $>140 \mathrm{mg} / \mathrm{dL})$ and D ( $\geq 1$ FPG $\geq 126 \mathrm{mg} / \mathrm{dL}$ ), which were too sensitive and had very low PPVs. Definitions $\mathrm{H}$ and $\mathrm{I}$, which include a combination of elevated RPG and FPG, had the highest PPVs and thus were least likely to falsely identify any patients as having DM. However, these definitions also missed more patients with DM.

\section{DISCUSSION}

In a large cohort of patients with AMI, we examined several potential combinations of random and fasting glucose levels obtained during hospital admission to identify patients with DM (defined by a core laboratory HbAlc $\geq 6.5 \%$ ). For DM screening, an RPG on arrival of $>140 \mathrm{mg} / \mathrm{dL}$ or an FPG $\geq 126 \mathrm{mg} / \mathrm{dL}$ were both highly sensitive for identifying patients with HbAlc-defined DM. In addition, a combination of RPG on arrival (using either the cut-point of $>140 \mathrm{mg} / \mathrm{dL}$ or $>180 \mathrm{mg}$ / $\mathrm{dL}$ ) or an FPG $\geq 126 \mathrm{mg} / \mathrm{dL}$ proposed as triggers for subsequent HbAlc testing, would lead to a marked reduction in the number of screening HbAlc tests 
performed (40-50\% of the AMI population) and would detect $82-86 \%$ of patients with incident DM. Alternatively, a single FPG $\geq 126 \mathrm{mg} / \mathrm{dL}$ to trigger HbA1c testing could also be used to identify the vast majority of patients with AMI with DM. Furthermore, the cohort of patients whose incident DM diagnosis would have been missed by such targeted HbAlc screening had relatively mild elevations of HbAlc; thereby potentially minimizing the impact of not recognizing these patients during their AMI hospitalizations. Finally, we identified several definitions with high accuracy that would be reasonable surrogates for the definition of DM in research studies, when HbAlc is not available.

Unrecognized DM is common in patients hospitalized with AMI. ${ }^{6}$ Accordingly, there have been efforts to diagnose DM during AMI hospitalization using oral glucose tolerance testing and HbA1c. ${ }^{10}{ }^{11}$ European guidelines recommend screening patients with AMI with an $\mathrm{HbAlc}$ and/or fasting glucose and, if inconclusive, an oral glucose tolerance test. ${ }^{16}$ However, given the acute fluctuations in blood glucose during AMI, ${ }^{17}$ it is unclear what thresholds of glucose necessitate further testing. Some researchers have sought to establish risk scores for diabetes based on waist circumference, family history, age, etc, ${ }^{18}$ but these have not been routinely implemented in patients with AMI and have not been recommended by guidelines due to increased complexity beyond simply testing HbAlc. We have established two potential strategies, using routinely collected admission and FPG levels, which could be used to inform selective screening of HbA1c. Given the ubiquitous use of electronic medical records in many countries, such a protocol could also be automated, with triggers to conduct a reflex add-on HbA1c if cardiac markers are elevated, admission RPG $>180$ (or $140 \mathrm{mg} / \mathrm{dL}$ ), or FPG $\geq 126 \mathrm{mg} / \mathrm{dL}$ (per morning chemistry panel), and no HbAlc measurement documented within the past 3 months. This information could not only improve the identification of new DM but would also be useful in monitoring the efficacy of glycaemic control in patients with treated DM. ${ }^{19}$ Importantly, these strategies were successful in identifying the vast majority of patients with DM while measuring HbAlc in only $40-50 \%$ of patients with AMI and reducing the number needed to screen by half. Universal HbA1c testing does have the advantage of also identifying the large proportion of patients with pre-DM (HbAlc 5.7-6.4\%) who may benefit from nutritional and lifestyle counseling, and thus this strategy may be appropriate for healthcare systems without substantial testing limitations. While an $\mathrm{HbAlc}$ test is not prohibitively expensive, the costs associated with 'universal' HbAlc screening of patients with AMI are not inconsequential in resource-limited healthcare settings, where these more selective strategies (based on data already collected) may be more cost-effective.

Furthermore, we have identified several combinations of fasting and admission glucose levels that could be used as potential surrogate definitions of DM when HbA1c is not available. These are particularly useful in large studies where HbA1c levels are missing in a substantial proportion of patients. In these cases, researchers may prefer one definition over another, depending on the goals of the study. For example, if a particular project needs to assure the DM group includes only patients with DM, then a definition with very high PPV, such as $\mathrm{H}$ or I, would be optimal. However, if a project wants to assure the non-DM group does not include any patients with DM, then a definition with highest NPV such as A, D, or F, would be optimal. For most projects, a balance between these is desired, which is where accuracy becomes most relevant.

There are potential limitations to our study that should be considered. First, although our study represents a large and diverse group of patients with AMI from academic and non-academic US hospitals that varied with respect to size and geography, it is unclear if our results would be generalizable to non-US patients with AMI. Second, patients admitted with high glucose levels or diet-controlled DM may have been empirically treated with correctional 'sliding scale' insulin during their AMI, which could potentially lower their subsequent FPG levels and thus interfere with our analyses of the relationship between FPG and HbAlc levels. However, typical correctional insulin protocols would not have impacted admission RPG levels and should have minimal (if any) impact on FPG-the two metrics evaluated in our study. Third, we used HbAlc $\geq 6.5 \%$ as the gold standard for DM in our study, which is known to be less sensitive than oral glucose tolerance testing. ${ }^{10}{ }^{11}$ However, it is emerging as a popular screening method for $\mathrm{DM}^{16}$ given its greater convenience in not requiring the patient to fast or complete an oral glucose tolerance test and since clinicians are accustomed to using this test for the management of DM once its presence is established. Finally, the current diagnostic criteria of the American Diabetes Association advise the confirmation of a high HbAlc with a second HbAlc test, ${ }^{14}$ which was not available in TRIUMPH. However, there were a subset of patients who had HbAlc tests drawn locally during the acute hospitalization, and $91 \%$ of these locally drawn HbA1c levels were also $\geq 6.5 \%$. In addition, we performed a sensitivity analysis among those with both values and found that the screening strategies remained highly effective.

In conclusion, in a large, multicenter cohort of patients with AMI, we identified two potential selective screening methods for DM using blood glucose levels collected as part of routine clinical care. These screening protocols required testing HbAlc in only $40-50 \%$ of patients with AMI and yet identified the vast majority of those with incident DM-an attractive strategy for clinical and economic reasons. We also found several definitions that could identify patients with probable DM based on routine blood glucose levels alone, which could be useful in large-scale clinical databases for 
research or population screening purposes when HbA1c levels are unavailable. Since patients with AMI and DM are at particularly high risk for recurrent cardiovascular events and the microvascular complications of DM, identification of patients with DM during AMI is important, so these individuals can be targeted for more intensive educational, lifestyle, or even medical interventions for their DM. Future work evaluating the implementation of screening processes and their impact on DM identification and management is needed.

\section{Author affiliations}

${ }^{1}$ Saint Luke's Mid America Heart Institute, University of Missouri-Kansas City, Kansas City, Missouri, USA

${ }^{2}$ Department of Endocrinology, Yale University School of Medicine, New Haven, Connecticut, USA

${ }^{3}$ Saint Luke's Mid America Heart Institute, Kansas City, Missouri, USA

${ }^{4}$ University of Texas Southwestern Medical Center, Dallas, Texas, USA

${ }^{5}$ Emory School of Medicine, Atlanta, Georgia, USA

${ }^{6}$ Saint Louis University, St. Louis, Missouri, USA

${ }^{7}$ University of Gothenburg, Gothenburg, Sweden

Contributors SVA designed the study and the statistical analysis plan, analyzed and interpreted the data, and drafted and revised the paper. KJL, SEI, and MK designed the study, interpreted the data, and drafted and revised the paper. YL analyzed and interpreted the data, and drafted and revised the paper. DKM, AG, JMS, ML, and JAS interpreted the data, and drafted and revised the paper.

Funding TRIUMPH was sponsored by a grant from the National Institutes of Health (National Heart, Lung, Blood Institute): SCCOR Grant \#P50HL077113-01. This study was sponsored by a research grant from Genentech, South San Francisco, California, USA. The funding organizations did not play a role in the conduct of the study or in the collection, management, analysis, and interpretation of the data.

Competing interests DKM received consultant honoraria from Takeda, Janssen, Merck, Regeneron, and Boehringer Ingelheim; and received clinical trial leadership honoraria from Boehringer Ingelheim, Takeda, Orexigen, Genentech, Roche, AstraZeneca, Bristol Myers Squibb, Eli Lilly, Daiichi Sankyo, Merck, Eisai, Omthera, Lexicon, Novo Nordisk, GlaxoSmithKline, and Janssen. JMS received grant support from GE Healthcare; consultant honoraria from Cordis Corp.; and serves on the speaker's bureau for Astra Zeneca, Astellas, and InfraReDx LLC. JAS received research grants from the NHLBI, American Heart Association, American College of Cardiology Foundation, Gilead, Lilly, EvaHeart, and Amorcyte; and received consultant honoraria from United Healthcare, Genentech, and Amgen. MK received research grants from the American Heart Association, Genetech, Sanofi-Aventis, Gilead, Medtronic Minimed, Glumetrics, Maquet, and Eisai; and received consultant honoraria from Genentech, Gilead, F Hoffmann LaRoche, Medtronic Minimed, AstraZeneca, Abbvie, Regeneron, Edwards Lifesciences, and Eli Lilly.

Patient consent Obtained.

Ethics approval Institutional Research Board at each institution in TRIUMPH.

Provenance and peer review Not commissioned; externally peer reviewed.

Data sharing statement No additional data are available.

Open Access This is an Open Access article distributed in accordance with the Creative Commons Attribution Non Commercial (CC BY-NC 4.0) license, which permits others to distribute, remix, adapt, build upon this work non- commercially, and license their derivative works on different terms, provided the original work is properly cited and the use is non-commercial. See: http:// creativecommons.org/licenses/by-nc/4.0/

\section{REFERENCES}

1. Bartnik M, Ryden L, Ferrari R, et al. The prevalence of abnormal glucose regulation in patients with coronary artery disease across Europe. The Euro Heart Survey on diabetes and the heart. Eur Heart J 2004;25:1880-90.

2. Gore MO, Patel MJ, Kosiborod M, et al. Diabetes mellitus and trends in hospital survival after myocardial infarction, 1994 to 2006: data from the national registry of myocardial infarction. Circ Cardiovasc Qual Outcomes 2012;5:791-7.

3. Haffner SM, Lehto S, Ronnemaa T, et al. Mortality from coronary heart disease in subjects with type 2 diabetes and in nondiabetic subjects with and without prior myocardial infarction. $N$ Engl J Med 1998;339:229-34.

4. Donahoe $\mathrm{SM}$, Stewart $\mathrm{GC}, \mathrm{McCabe} \mathrm{CH}$, et al. Diabetes and mortality following acute coronary syndromes. JAMA 2007;298:765-75.

5. Norhammar A, Tenerz A, Nilsson G, et al. Glucose metabolism in patients with acute myocardial infarction and no previous diagnosis of diabetes mellitus: a prospective study. Lancet 2002;359:2140-4.

6. Lankisch M, Futh $\mathrm{R}$, Schotes $\mathrm{D}$, et al. High prevalence of undiagnosed impaired glucose regulation and diabetes mellitus in patients scheduled for an elective coronary angiography. Clin Res Cardiol 2006;95:80-7.

7. Fonarow GC, Gawlinski A, Moughrabi S, et al. Improved treatment of coronary heart disease by implementation of a Cardiac Hospitalization Atherosclerosis Management Program (CHAMP). Am J Cardiol 2001;87:819-22.

8. Lappe JM, Muhlestein JB, Lappe DL, et al. Improvements in 1-yea cardiovascular clinical outcomes associated with a hospital-based discharge medication program. Ann Intern Med 2004;141:446-53.

9. Capes SE, Hunt D, Malmberg K, et al. Stress hyperglycaemia and increased risk of death after myocardial infarction in patients with and without diabetes: a systematic overview. Lancet 2000;355:773-8.

10. de Mulder M, Oemrawsingh RM, Stam F, et al. Comparison of diagnostic criteria to detect undiagnosed diabetes in hyperglycaemic patients with acute coronary syndrome. Heart 2012;98:37-41.

11. Bartnik M, Ryden L, Malmberg K, et al. Oral glucose tolerance test is needed for appropriate classification of glucose regulation in patients with coronary artery disease: a report from the Euro Heart Survey on Diabetes and the Heart. Heart 2007;93:72-7.

12. Bonora $\mathrm{E}$, Tuomilehto $\mathrm{J}$. The pros and cons of diagnosing diabetes with A1C. Diabetes Care 2011;34(Suppl 2):S184-90.

13. Arnold SV, Chan PS, Jones PG, et al. Translational Research Investigating Underlying Disparities in Acute Myocardial Infarction Patients' Health Status (TRIUMPH): Design and Rationale of a Prospective Multicenter Registry. Circ Cardiovasc Qual Outcomes 2011;4:467-76.

14. American Diabetes Association. Diagnosis and classification of diabetes mellitus. Diabetes Care 2010;33(Suppl 1):S62-9.

15. Deedwania P, Kosiborod M, Barrett E, et al. Hyperglycemia and acute coronary syndrome: a scientific statement from the American Heart Association Diabetes Committee of the Council on Nutrition, Physical Activity, and Metabolism. Anesthesiology 2008;109:14-24.

16. Ryden L, Grant PJ, Anker SD, et al. ESC Guidelines on diabetes, pre-diabetes, and cardiovascular diseases developed in collaboration with the EASD: the Task Force on diabetes, pre-diabetes, and cardiovascular diseases of the European Society of Cardiology (ESC) and developed in collaboration with the European Association for the Study of Diabetes (EASD). Eur Heart $J$ 2013;34:3035-87.

17. Bellodi G, Manicardi V, Malavasi V, et al. Hyperglycemia and prognosis of acute myocardial infarction in patients without diabetes mellitus. Am J Cardiol 1989;64:885-8.

18. Lindstrom J, Tuomilehto J. The diabetes risk score: a practical tool to predict type 2 diabetes risk. Diabetes Care 2003;26:725-31.

19. Stolker JM, Sun D, Conaway DG, et al. Importance of measuring glycosylated hemoglobin in patients with myocardial infarction and known diabetes mellitus. Am J Cardiol 2010;105:1090-4. 